\title{
Proliferation Changes in Dentate Gyrus of Hippocampus during the First Week following Kainic Acid-induced Seizures
}

\author{
Peifei Gu, ${ }^{*},{ }^{a}$ Yue LI, ${ }^{b}$ Yu SHAng, ${ }^{c}$ Yue Hou, ${ }^{d}$ and Songyan $\mathrm{ZHAO}^{d}$ \\ ${ }^{a}$ Centre of Clinical Pharmacology, Northeast Pharmaceutical Science and Technology Co., Ltd. \\ Shenyang 110027, P. R. China, ${ }^{b}$ Department of Anesthesiology, The First Affiliated Hospital \\ of Harbin Medical University, Harbin 150001, P. R. China, 'Department of Anesthesia, \\ PLA463 Hospital, Shenyang 110042, P. R. China, and dDivision of Pharmacology, \\ Shenyang Pharmaceutical University, Shenyang 110016, P. R. China
}

(Received July 2, 2010; Accepted September 9, 2010)

\begin{abstract}
Long-term neuroplastic changes in dentate gyrus (DG) have been reported after seizure induction and were shown to contribute to epitogenesis of epilepsy. These changes include increased number of newborn granule cells, sprouted mossy fibers, granule cell layer dispersion, etc. The aim of current study is to determine the acute progression of neuroplastic changes involved newly generated granule cells after kainic acid (KA) -induced seizures. Doublecortion (DCX) analysis was used to examine the newly generated granule cells morphology 1-7 days after seizure induction. Quantitative analysis of DCX-labeled cells at different times shows that there are some rapid changes in the dentate gyrus. At day 7 epileptical mice induced an increase of the number of DCX-labeled cells in DG. At days 3 and 7 after epilepsy induction, the percentage of DCX-labeled cells per DG were significantly increased. These results show that seizures are capable to increase the number of new granule cell within a short time for function activation in post-seizure period. Therefore, the rapid changes in the DG might be having a potential for hippocampus neuroplastic function.
\end{abstract}

Key words_ — kainic acid; epilepsy; dentate gyrus; doublecortion

\section{INTRODUCTION}

Epilepsy is a common chronic neurological disorder that is characterized by recurrent unprovoked epileptic seizures. Epilepsy is usually controlled, but not cured, with medication, even though by surgery. Epilepsy should not be understood as a single disorder, but rather as a group of syndromes with vastly divergent symptoms but all involving episodic abnormal electrical activity in the brain., ${ }^{12)}$ Among other pathological findings, an abnormal broadening of the dentate gyrus in epileptic hippocampus is often observable but up to date it remains unclear if this morphological change represents a consequence or a possible cause of epilepsy. Not only mature neurons are subject to alterations in the epileptic hippocampus with seizure-activity, also the activity strongly induces cell proliferation and net neurogenesis in rodent epilepsy models. ${ }^{3,4)}$ Studies of adult rodent models of limbic epileptogenesis or acute seizures showed that seizure or seizure-induced injury stimulates neurogenesis in both the SGZ and SVZ of hippocampus. ${ }^{4)}$ In the dentate gurys, epilepsy increases the proliferation

\footnotetext{
*e-mail: gpeifei9999@ hotmail.com
}

of progenitors five- to ten-fold after a latent period.5) Most of these newborn cells differentiate into granule neurons, some of which mislocate in the hilus region. Because of proliferation association with neurogenesis processes, there has been recently interested in doublecortin (DCX) as a candidate marker for neurogenesis. ${ }^{3-5)}$

Researches suggested that the number of DCX-expressing cells correlates with the level of cellular proliferation in the dentate gyrus, ${ }^{6}$ ) however, it remains unclear that whether this correlation is maintained when levels of neurogenesis are modulated by external factors, such as seizures. Recently findings indicate that seizures induced by various experimental manipulations (kindling, pilocarpine, kainic acid) increase neurogenesis in the adult rodent DG. ${ }^{7)}$ Previous studies showed that newly generated neurons significant increase at least 2 weeks after kainic acid (KA) induced seizures, it is unclear whether these neuroplastic changes happened during the shorter and acuter time-points after KA induced seizures.

Therefore we tried to answer the following question in this study: whether the newly generated neuronal proliferation was already appeared during the first week post-seizure by DCX staining. 


\section{MATERIALS AND METHODS}

Animals All experimental C57/BL6 mice were purchased from Shenyang, China. After they arrived, weighing 20-25 g that were housed in individual cages on $12 \mathrm{~h} \mathrm{light/dark} \mathrm{schedules} \mathrm{with} \mathrm{water} \mathrm{and} \mathrm{food}$ were available ad libitum. Animal procedures were approved by the Veterinary Office of China. All efforts were made to minimize animal suffering and to reduce the number of animals used.

Chemicals DCX antibody and Alexa Fluor 594 donkey-anti-goat antibody were purchased from Santa Cruz Biotechology (USA). Hoechst 33258 were purchased from Molecular Probes (Eugene, OR). Kainic acid and other chemicals were purchased from Sigma Chemicals (St. Louis, MO).

Kainic Acid Treatment and Seizure Evaluation

KA was administrated to induce epilepsy in mice. Kainic acid was dissolved in isotonic saline ( $\mathrm{pH} 7.3-$ 7.4) at a concentration of $24 \mathrm{mg} / \mathrm{kg}$ or $6 \mathrm{mg} / \mathrm{kg}$ in 10 $\mathrm{mg} / \mathrm{ml}$ volume. Mice were separated into individual cages and given i.p. injections of KA, the initial dose was $24 \mathrm{mg} / \mathrm{kg}$, intraperitoneally. After that, when necessary, mice were given repeat doses of $6 \mathrm{mg} / \mathrm{kg}$ every 30 min until animals had jumping clonus. Seizure intensity was evaluated according to six-point seizure scale for mice ${ }^{8)}$ which adapted from a fivepoint scale for rat.9) Control animals were injected with isotonic saline intraperitoneally.

Tissue Preparation 1, 3, 7 Days following seizure induction, mice were deeply anaesthetized with Nembutal $(100 \mathrm{mg} / \mathrm{kg}$ i.p) and then transcardially perfused with $0.1 \mathrm{M}$ PBS (pH 7.4) followed by 4\% paraformaldehyde in $0.1 \mathrm{M}$ PBS ( $\mathrm{pH}$ 7.4). The brains were removed from the skull, and placed in the same fixative for $4 \mathrm{~h}$. After post fixation, the brains were cryoprotected in $30 \%$ sucrose, and serial frozen coronal sections $(20 \mu \mathrm{m})$ were cut on cryostat. The following immunoflorescent procedures were used: doublecortin (DCX) staining to estimate the newborn cells.

Immunofluorescent Staining For DCX fluorescent staining, sections were used then washed three times in PBS and serum with $0.3 \%$ TritionX-100 in PBS was applied for one hour for blocking step and subsequent DCX (1:500, goat polyclonal) primary antibody was used. The sections were then rinsed for $20 \mathrm{~min}$ in PBS and incubated with corresponding secondary antibodies: Alexa Fluor 594 donkey-anti- goat $(1: 1000)$ in $0.3 \%$ TX-PBS for $2 \mathrm{~h}$. Then, sections were rinsed according to routine method, washing, Hoechst staining, and coverslips.

In fluorescent staining, nuclei were stained with Hoechst 33258 (Molecular Probes, Eugene, OR). Sections were mounted out of PBS and coverslipped with Permount. Control experiments include the incubation of sections in the absence of primary antibodies. Signals were subsequently analyzed under appropriate filter using Leica DM 6000 fluorescent microscope (Germany). All images were captured using a Leica camera or Nikon CCD camera (Japan) .

Statistical Analysis Sections between -1.5 and $-2.8 \mathrm{~mm}$ to bregma were used in this experiment. To determine the mean grey values of different staining in dentate gyrus, every three areas of one section and three randomly chosen sections for each mouse were selected. The reference grey value was determined on no-staining area. All data in this study are presented as mean \pm S.E.M. Cell counted were averaged for each mouse and were compared among treatment groups using one-way ANOVA followed by a LSD post-hoc test; ${ }^{*} p<0.05,{ }^{* *} p<0.01$. (SPSS 13.0 software).

\section{RESULTS AND DISCUSSION}

Seizure Scoring Forty mice were used in this experiment; 25 to establish KA model, 15 mice with saline injection. All KA-treated animals displayed behavior seizures. According to the method mentioned above, after 5 times injection $(30 \mathrm{mg} / \mathrm{kg}), 9(36 \%)$ mice displayed progressive seizures over the next 30 min. After 6 times $(36 \mathrm{mg} / \mathrm{kg})$ injection, $10(40 \%)$ treated animals displayed progressive seizures over the next $30 \mathrm{~min}$. After 7 times injection, 6 (24\%) treated animals of left displayed progressive seizures over the next $30 \mathrm{~min}$. All animals displayed severe seizure, and 2 mice were dead during the epilepsy duration and one mouse dead following KA injection. The average dose to induce seizure is $34.64 \mathrm{mg} / \mathrm{kg}$. No seizure activity was observed in animals treated with saline.

The Morphology and Distribution of the DCX-labeled Cells According to Fig. 1, two parameters were examined for each time points following KA-induced seizure, which include the number of DCXlabels cells in DG and the percentage of DCX-labeled cells per DG. Only significant differences in these two parameters are reported for each time point. The total 

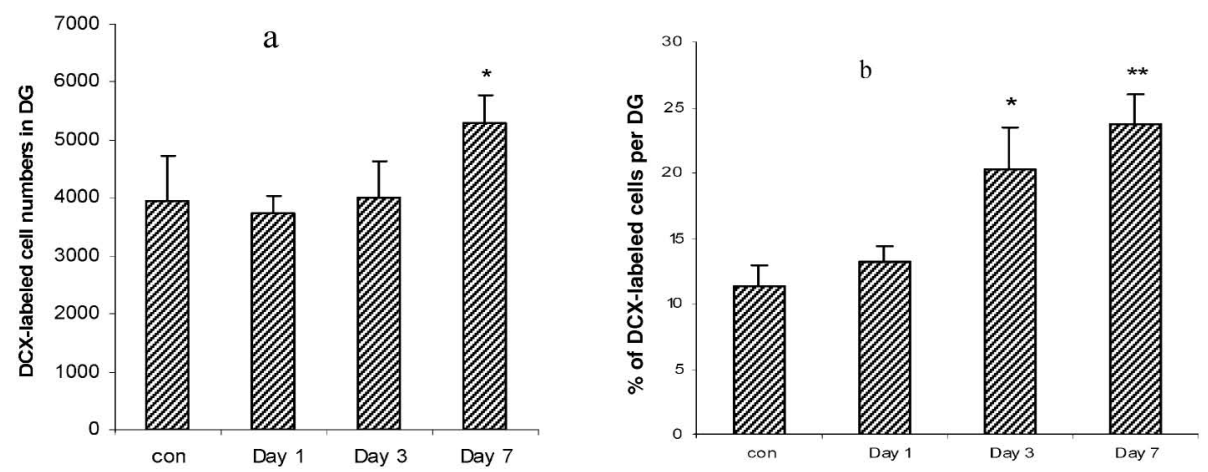

Fig. 1. Histograms of the Numbers of DCX-labeled Cells (a) and the Percentage of DCX-labeled Cells (b) in DG All data are showed as mean \pm S.E.M. ${ }^{*} p<0.05,{ }^{* *} p<0.01$ compared with control group ( $n=5-6$ animals per group).

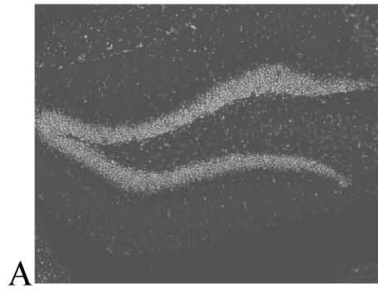

$\mathrm{b}$
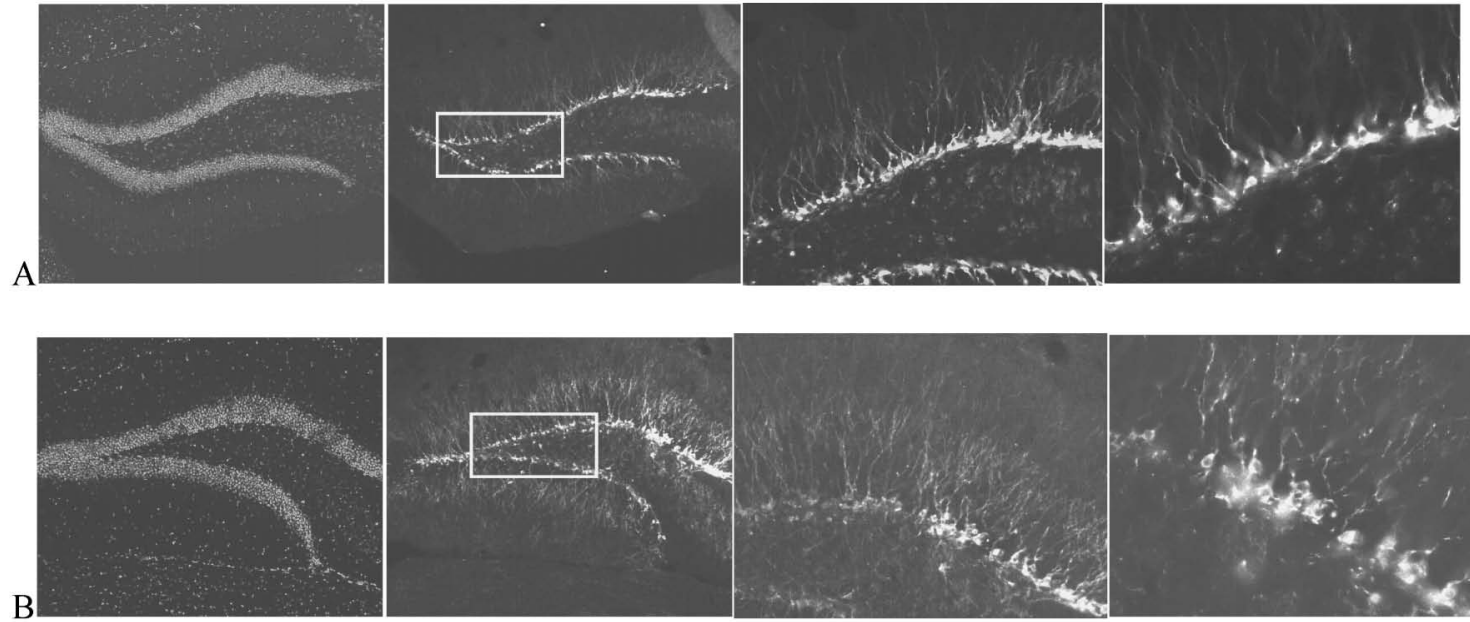
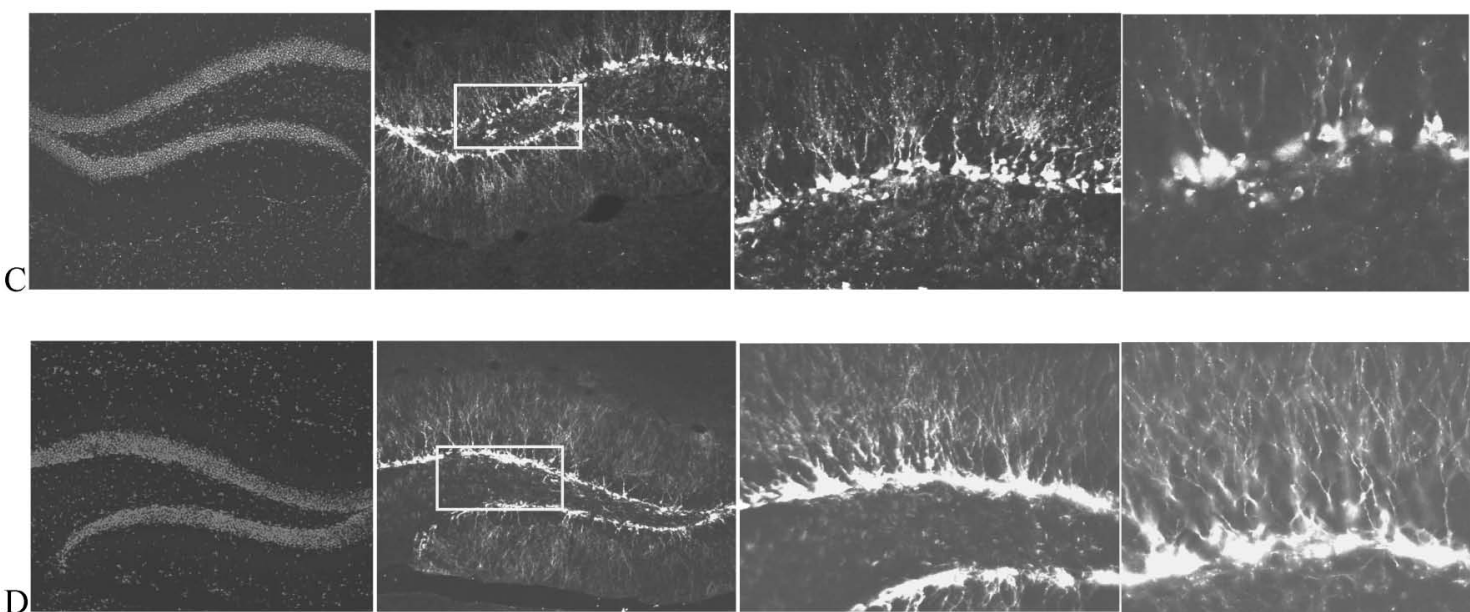

Fig. 2. Morphology of DCX Expression in the DG of Hippocampus at Different Time Points

A: control; B: $24 \mathrm{~h}$ after KA model established; C: $72 \mathrm{~h}$ after KA model established; D: 7 days after KA model established. a: Hoechst staining (100 $\times)$; b: DCX fluorescent staining $(100 \times)$; c: DCX fluorescent staining $(200 \times)$; d: DCX fluorescent staining $(400 \times)$.

number of DCX-positive cells per DG in control group is $3954 \pm 758$; and the percentage of DCX-labeled cells per DG in control mice is $11.31 \pm 1.62$. At day 1 after KA-induced seizures, the number of DCXlabels cells in DG and the percentage of DCX-labeled cells per DG did not show significant difference com- 
pared with control group. At day 3 afte seizures, the percentage of DCX-labeled cells per DG was increased to $20.27 \pm 3.18$, which has a significant increase compared with control group (Fig. 1, $p<$ $0.05)$. At day 7 after seizures, both parameters appeared significant increased compared with control group; the percentage of DCX-labeled cells per DG was increased to $23.75 \pm 2.30$ (Fig. $1, p<0.01$ ); and the number of DCX-labels cells in DG was increased to $5284 \pm 500$ (Fig. $1, p<0.05$ ).

The current study demonstrates several rapid neuroplastic changes involving newly generated granule cells during the first 7 days after KA-induced seizures. The earliest change observed from the parameters analyzed indicate that, at day 1 after KA-induced seizures, there was an increase in the percentage of DCX-labeled cells with basal dendrites and an increase in the progenitor cell population. At day 3 after seizures, the thickness of the DCX-labeled cell layer was increased (Fig. 2). At day 3 after seizures, the number of DCX-labeled cells was increased. DCXpositive cells were typically found in the SGZ, at the border between the SGZ and the granule layer (GL), or in the GL. Consistent with previous studies, epileptic mice have a great number of new-born neurons. Basal dendrites were observed on DCX-labeled neurons for both control and epileptic mice (Fig. 2). In the research, morphological analysis of the DCX immunolabeled cells with basal dendrites revealed that the new born neurons with basal dendrites have a significantly increase in the epileptic mice compared with control after day 3 (Fig. 2). These changes using KAinduced seizures are consistent with the results from a previous study showing that DCX-labeled cells may become proliferate in response to KA-induced seizures. ${ }^{10)}$ The fact that many of the progenitor cells were found in dense clusters beyond the hilar border by 3 days after seizures suggests that they may also contribute to the thickening of the newly born granule cell layer, because they are generating cells beyond the hilar border region. This neuroplastic change is analogous to human and primate TLE and rodent models of TLE, where granule cell layer dispersion and hilar ectopic granule cells are prominent features of the epileptic hippocampus. ${ }^{11,12}$ These new-born granule cells have been shown to be integrated into granule cell circuitry functionally and develop protective effect in epileptic activity, ${ }^{12,13)}$ but in this research its specific significance still needs further elucidation.

\section{REFERENCES}

1) Houser C. R., Brain Res., 535, 195-204 (1990).

2) Helmstaedter C., Prog. Brain Res., 135, 439453 (2002).

3) Bengzon J., Kokaia Z., Elmer E., Nanobashvili A., Kokaia M., Lindvall O., Proc. Natl. Acad. Sci. USA., 94, 10432-10437 (1997).

4) Parent J. M., Yu T. W., Leibowitz R. T., Geschwind D. H., Sloviter R. S., Lowenstein D. H., J. Neurosci., 17, 3727-3738 (1997) .

5) Parent J. M., Janumpalli S., McNamara J. O., Lowenstein D. H., Neurosci. Lett., 247, 912 (1998).

6) Brown J. T., Gill C. H., Farmer C. E., Lanneau C., Randall A. D., Pangalos M. N., Collingridge G. L., Davies C. H., Epilepsy Res., 57, 121-136 (2003).

7) Shapiro L. A., Ribak C. E., Brain Res. Rev., 48, 43-56 (2005).

8) Kempermann G., Kuhn H. G., Gage F. H., Proc. Natl. Acad. Sci. USA., 94, 10409-10414 (1997).

9) Racine R. J., Electroencephalogr. Clin. Neurophysiol., 32, 281-294 (1972).

10) Jessberger S., Romer B., Babu H., Kempermann G., Exp. Neurol., 196, 342-351 (2005) .

11) Scharfman H., Goodman J., Macleod A., Phani S., Antonelli C., Croll S., Exp. Neurol., 192, 348-356 (2005).

12) Scharfman H. E., Gray W. P., Epilepsia., 48, 33-34 (2007).

13) Parent J. M., von dem Bussche N., Lowenstein D. H., Hippocampus, 16, 321-328 (2006). 